\title{
Actitudes de los estudiantes de origen inmigrante hacia una lengua minoritaria en España
}

\author{
Isabel Hevia Artime, Xosé Antón González Riaño \\ y Patricia Albá García
}

El ámbito escolar constituye un contexto privilegiado para la realización de estudios sociolingüísticos al incorporar características definitorias de la sociedad a la que se adscribe. En este sentido, la presente investigación recoge los resultados de un estudio que analiza las actitudes lingüísticas de un grupo de 123 escolares hacia la lengua tradicional y minoritaria del Principado de Asturias (lengua asturiana o asturiano) así como hacia la lengua oficial (castellano). A través de un cuestionario se constata que estos jóvenes manifiestan una actitud positiva hacia ambas lenguas, encontrándose diferencias actitudinales en función del origen del alumnado y otras variables sociolingüísticas.

Palabras claves: Lengua minoritaria, actitudes lingüísticas, diversidad cultural, alumnado inmigrante, asturiano.

Attitudes of students of immigrant origin to a minority language in Spain. The school environment is a privileged area for conducting sociolinguistic studies, mainly since it incorporates the characteristics of the society to which it belongs. In this sense, this research includes the results of a study that analyzes the linguistic attitudes of a group of 123 school children towards the traditional and minority language of the Principality of Asturias (Asturian or Asturian language) as well as the official language (Spanish). Through a questionnaire it is found that these young people manifest a positive attitude towards both languages, finding attitudinal differences according to the origin of the students and other sociolinguistic variables.

Keywords: minority language, linguistics attitudes, cultural diversity, immigrant students, Asturian.

\section{Introducción}

El presente estudio se centra en conocer las actitudes lingüísticas de los alumnos inmigrantes y autóctonos con respecto a las dos lenguas habla- 
das en el Principado de Asturias. ${ }^{1}$ Las lenguas presentes en esta comunidad son el castellano (lengua oficial en toda España) y la lengua asturiana o asturiano (lengua minoritaria y regional). La lengua asturiana es una de las lenguas romances que históricamente se formaron en la Península Ibérica a partir de la evolución del latín, junto al gallego, el portugués, el castellano, el catalán o el aragonés. Desde los años 70 se han llevado a cabo varias encuestas sociolingüísticas que vienen a indicar que en Asturias hablarían la lengua asturiana cerca de un 30\% de la población. De la última encuesta sociolingüística realizada por LleraRamo (2017), se desprende que un $65 \%$ de la población afirma que el asturiano es el habla vernácula de Asturias. Sin embargo, es una lengua minoritaria dentro de esta región que, según los informes del Atlas of the World's Languages in Danger of Disappearing de la UNESCO, está clasificada como «lengua en peligro». Ello tiene que ver con la falta de reconocimiento como lengua oficial de la comunidad y el hecho de que se la asocie con un status social inferior.

Como lengua materna empleada por una parte de su población, la lengua asturiana se mantiene no solo de una generación a otra, sino que también es posible adquirirla a través de procesos educativos formales y no formales. De hecho, la Ley de Uso del Asturiano de 1998 establece el aprendizaje voluntario de la lengua local (asturiano) en todas las etapas educativas no universitarias, si bien el castellano es en todos los casos la lengua vehicular de enseñanza. Es decir, la única presencia escolar del asturiano se circunscribe a la asignatura de 'Lengua y Literatura Asturiana'.

Por otro lado, Asturias es una región que ha sufrido la crisis de la sociedad industrial coincidiendo con la emergencia de la sociedad de la información. Las reconversiones industriales (siderurgia, minería, industria naval, etc.) han modificado profundamente las características de una sociedad que hasta los años 80 tenía un gran dinamismo social, económico y cultural. En los años 90 la población asturiana empieza a reducirse, y se detectan dos tendencias complementarias: aumento del envejecimiento de la población y reducción de la tasa de natalidad (ICSLRA 2004). Esta reducción de la población se ve atemperada en las dos últimas décadas por un importante aumento de la inmigración extranjera -con una importante presencia de inmigrantes de Europa del Esteque representaba en el 2017 el 3,9\% de la población. Aunque la presencia de personas inmigrantes es mucho menor que en otras zonas de España como Catalunya, Andalucía, Madrid o el Levante, nos encontramos en un contexto migratorio donde la lengua se convierte en elemento de inclusión y de exclusión en relación con la sociedad de acogida (Sorolla 2011), aspecto este complejo por desarrollarse en una comunidad en donde se produce un bilingüismo socialmente desequilibrado.

Lengua y migración / Language and Migration 12:2 (2020), 87-108

Edición impresa: ISSN 1889-5425. Edición en línea: ISSN 2660-7166. (C) Universidad de Alcalá 
En relación con la presencia del alumnado inmigrante en el sistema educativo asturiano, cabe destacar que este último grupo es minoritario, en el curso 2015/2016 suponía el 4,18\% del alumnado matriculado en Educación Primaria, entre los que destaca el alumnado procedente de Rumania (418 estudiantes), Marruecos (158 estudiantes) y Republica Dominicana (102 estudiantes). Sin embargo, la participación de este grupo en el sistema educativo empieza a percibirse como un fenómeno emergente y especialmente interesante, puesto que implica la diversidad en un contexto educativo en el que tradicionalmente ha prevalecido la uniformidad étnica del alumnado. En nuestra investigación centramos la atención en el alumnado de $6^{\circ}$ curso de Educación Primaria (12 años de edad), nivel educativo que cuenta con un alto número de alumnado inmigrante escolarizado en clases de lengua asturiana. Conocer las actitudes lingüísticas de este alumnado (y compararla con las del alumnado autóctono) resulta interesante y novedoso en esta comunidad, pues la actitud de los hablantes jóvenes hacia esta lengua minoritaria es determinante para difundirla en su medio social (Paris 2011; Pérez 2016), sobre todo teniendo en cuenta la precariedad con la que la lengua regional se instala en el sistema educativo.

En este contexto, se plantea el presente trabajo cuyos objetivos principales son los siguientes: a) Estudiar las actitudes lingüísticas hacia el asturiano y el castellano de una muestra de alumnos (autóctonos e inmigrantes) de $6 .^{\circ}$ curso de Educación Primaria (12 años) que asisten, o no, a clases de Lengua Asturiana; b) Comprobar si se produce un cambio de actitud hacia la lengua asturiana en función de distintas variables socioeducativas (sexo, procedencia del alumnado, condición lingüística familiar y asistencia o no a clases de asturiano).

\section{Algunos aspectos teóricos sobre las actitudes lingüísticas}

Como se sabe, en cualquier comunidad, sociedad o grupo humano sus miembros manifiestan, habitualmente, valoraciones con respecto al uso de las lenguas. Tales valoraciones no son neutras generalmente, sino que se caracterizan por manifestar juicios de valor (positivos o negativos) hacia las lenguas o variedades lingüísticas; a tales juicios se les denomina 'creencias sociolingüísticas' (García-Marcos 2015). Dentro de las creencias sociolingüísticas aparecen como una de sus principales las 'actitudes lingüísticas'. La actitud es la aceptación o rechazo de un hecho lingüístico que se produce por las creencias (favorables o adversas) hacia el hecho en cuestión. El término genérico de 'actitud' comienza a ser uti- 
lizado por Spencer (1862) a finales del siglo XIX y posteriormente se adscribe al ámbito de la psicología social, entendida esta como la ciencia que tiene por objeto entender y explicar el modo en que los pensamientos, sentimientos, comportamientos, etc. de las personas son influidos por el contexto social (Allport 1935). Oskamp (1991), por su parte, atribuye el éxito del término a la facilidad con la que la gente relaciona el concepto de actitud con el comportamiento de las personas en sociedad.

Es preciso subrayar, además, que las actitudes lingüísticas toman como referencia a las lenguas (o variedades lingüísticas) y, en consecuencia, constituyen una parte fundamental de la sociolingüística; es decir, de la disciplina que estudia las relaciones entre lengua y sociedad. Baker (1992) nos dice que esas actitudes se forman, se desarrollan y, en su caso, se modifican a través de la interacción de atributos individuales y las situaciones sociales. Por tanto, a la hora de estudiar las actitudes lingüísticas se hace necesario adoptar un enfoque psicosocial (Janés 2006), puesto que las actitudes lingüísticas no solo tienen como fin la valoración de unos determinados usos lingüísticos, sino también de sus hablantes. Cuando hablamos de lenguas minoritarias, son más bien las actitudes hacia sus hablantes las que hacen que estos sean considerados culturalmente inferiores. En definitiva, tal como afirma Trudgill (1985), las actitudes hacia cualquier lengua o dialecto no estándar son un reflejo de las estructuras de la sociedad. En el caso de las personas inmigrantes, las actitudes lingüísticas se revelan como reflejo de las actitudes sociales hacia el propio grupo y hacia la comunidad de acogida y, por tanto, es necesario conocerlas para entender el proceso de integración sociolingüística de los inmigrantes (Sancho 2013).

Tradicionalmente, se ha atribuido a las actitudes lingüísticas tres componentes básicos: a) perceptivo o cognitivo: creencias o valores que tiene un individuo ante una determinada lengua o variedad; b) afectivo o sentimental: sentimientos o emociones en relación con la forma lingüística considerada; y c) comportamental o conativo: reacciones concretas ante el uso de la lengua y la presencia de sus hablantes (Breckler 1984; Zanna y Rempel 1988; López-Morales 1989; Moreno 1998). Sin embargo, existe una falta de consenso acerca de los componentes que constituyen las actitudes. Dependiendo de la tendencia teórica, algunos autores (Almeida y Vidal 1995) consideran que la actitud lingüística estaría dominada por el rasgo conativo, pues la percepción junto con los pensamientos positivos o negativos que inspira la lengua provocaría determinadas actitudes que, finalmente, afectarían la actuación lingüística. Mientras que autores como Ibáñez (2004) manifiestan que solo podemos hablar de actitud cuando hay un compromiso personal, dando mayor peso al componente afectivo.

Por su parte, en el contexto español, el número de estudios de investigación sobre actitudes lingüísticas entre la población estudiantil inmi- 
grante es más bien limitado, debido a que la inmigración a gran escala es un fenómeno bastante reciente. Sin embargo, debe recordarse que las actitudes juegan un papel primordial en el aprendizaje de la lengua, como se refleja en todos los modelos destinados a analizar el logro final en el proceso de aprendizaje L2 (Gardner y MacIntyre 1993; Tremblay y Gardner 1995). El mantenimiento de la motivación de los estudiantes requiere algún tipo de apoyo afectivo y esta parece ser una función de las actitudes. Creemos que en contextos donde coexisten lenguas minoritarias y mayoritarias, el idioma extranjero (inglés) y los propios idiomas de los inmigrantes, requieren el examen de las actitudes lingüísticas. Es claro, pues, que, si las autoridades educativas tienen la intención de implementar políticas lingüísticas coherentes, es necesario investigar las actitudes lingüísticas de los estudiantes locales e inmigrantes.

En el plano de las actitudes lingüísticas, por otra parte, las manifestaciones más negativas se conocen como 'prejuicio lingüístico'. El prejuicio lingüístico hace referencia a una actitud irracional y fuertemente negativa ante una lengua y sus hablantes (Tusón 1997). De hecho, las personas tienden a desconfiar de quienes hablan de forma diferente, dando por sentado que la forma de hablar de uno es la más natural (Wallwork 1985). Por otro lado, en sociolingüística también se viene estudiando lo que se llama 'deslealtad lingüística', entendida como la actitud de aquellos hablantes que se avergüenzan de su propia lengua, que rechazan usarla y que llegan, incluso, a negarla (González 2002). Es preciso añadir que, aunque generalmente se admite que las actitudes parecen configurarse en torno a la adolescencia (Perlman y Cozby 1985; Baker 1992), se ha demostrado que incluso niños de 5 años pueden manifestar prejuicios lingüísticos y ello a pesar de que se trate de lenguas tan prestigiosas como el inglés o el francés (Schneiderman 1976). De hecho, los juicios de valor basados en el habla poseen una relación muy estrecha con el proceso educativo. La escuela se convierte en un espacio de transmisión e imposición de la lengua culta o estándar, mientras que las otras formas de expresión y comunicación lingüísticas diferentes son consideradas a menudo registros inferiores (Lewandowski 1982; Milroy 2007).

Por otro lado, en los estudios sociolingüísticos que tienen en cuenta la variable de sexo, se encuentra que las mujeres, por lo general, suelen manifestar en mayor medida que los hombres una predilección por las variantes estándar. Por tanto, tienden a usar las formas prestigiosas con más frecuencia que los hombres de igual estatus socio-económico. Según Trudgill (1992) y Trudgill y Hernández (2007) estas tendencias que caracterizan el comportamiento lingüístico hay que relacionarlas con la idea de 'prestigio', entendido este como un comportamiento lingüístico motivado por las actitudes sociales ante el uso de determinas formas lingüísticas. Profundizando en lo anterior, los citados autores se 
refieren a la idea de 'prestigio manifiesto o abierto' en referencia al respeto manifestado ante el uso de formas lingüísticas consideradas socialmente como prestigiosas, mientras que reservan el concepto de 'prestigio encubierto' para referirse a usos lingǘsticos que, pese a no tener tal prestigio social, sí permiten identificaciones afectivo-culturales relevantes para algunos miembros de la comunidad lingüística en función de motivaciones subjetivas o emocionales. Asociando tales conceptos a la práctica lingüística de hombres y mujeres, la sociolingüística viene atribuyendo, en general y como se ha dicho, a las mujeres una conducta conservadora que favorecería el uso público de variables lingüísticas más prestigiosas (prestigio manifiesto), mientras que reservaría la idea de prestigio encubierto para distinguir las valoraciones favorables que las formas no estándares, de bajo estatus social o 'incorrectas' tendrían como connotaciones de masculinidad. No obstante, lo anterior, Labov (1966) pone de manifiesto que en algunos casos las mujeres tienen en cuenta otras consideraciones para asumir normas de prestigio encubierto relacionadas, por ejemplo, con la simpatía y lealtad ante el uso de determinadas formas lingüísticas de la comunidad y ello al margen de su valoración socio-cultural. Serrano (2008) por su parte y profundizando en lo anterior, manifiesta que las mujeres de diferentes comunidades dan respuestas distintas y concluye que en las sociedades americana e inglesa la mujer suele mostrar más conservadurismo, mientras que en las sociedades europeas suelen ser más innovadoras y en algunas sociedades africanas muestran un doble comportamiento, conservador e innovador a la vez.

Para concluir este apartado, nos gustaría resaltar que, en el caso de las personas inmigrantes, las actitudes lingüísticas que presentan hacia la lengua o las lenguas de la sociedad de acogida pueden incidir de manera directa sobre su proceso de integración sociolingüística y socioeducativa. Hay que tener en cuenta, tal como señala Sancho (2013), que en España nos encontramos con una compleja situación lingüística en donde existen zonas de bilingüismo (castellano y lengua de la comunidad) y zonas de gran uniformidad en torno al castellano.

\section{El estudio de las actitudes lingüísticas en el contexto educativo}

El estudio de las actitudes lingüísticas en el marco escolar es especialmente relevante habida cuenta de la relación entre actitudes lingüísticas y adquisición de una segunda lengua, reto al que se enfrenta la mayoría del alumnado recién llegado. Tal relevancia ha sido puesta de manifiesto desde décadas atrás en el contexto internacional (Hamers y Blanc 1983; 
Appel y Muysken 1996; Lasagabaster 2003; Masgoret y Gardner 2003; Huguet y González 2004; Esteban y Chaib 2014).

Dada la temática de nuestro estudio, algunas investigaciones han evidenciado que es posible favorecer actitudes lingüísticas positivas hacia las lenguas minoritarias. Tal es el caso de los resultados registrados en el grupo de niños de la tribu Inuit en Québec (Wright y Bougie 2007) que mantienen actitudes lingüísticas de fidelidad porque consideran la lengua como un importante elemento de identidad. De hecho, las actitudes hacia las minorías étnico-lingüísticas mejoran, incluso en el grupo social mayoritario, cuando se ponen en práctica medidas de educación intercultural que tengan en cuenta a la lengua minoritaria (Verkuyten y Thijs 2010). Es preciso señalar, finalmente, que los efectos de las actitudes positivas hacia las lenguas minoritarias se ven fuertemente incrementados cuando desde las instituciones y los gobiernos se ponen en marcha medidas efectivas de planificación y normalización lingüística (Moriarty 2010).

Por lo que respecta al ámbito español, Cataluña es la comunidad que más investigación genera en torno a las actitudes lingüísticas entre los escolares. En este sentido son interesantes los trabajos pioneros de Huguet y Suils (1998), Huguet (2001) y Huguet y Llurda (2001) en los que se comparan las actitudes lingüísticas de los escolares de educación secundaria en Cataluña y en la zona catalanoparlante de Aragón. Los resultados de estos estudios ponen en evidencia la importancia de las medidas institucionales de normalización lingüística a la hora de generar cambios actitudinales en la población escolar y, como consecuencia, en la mejora del rendimiento lingüístico escolar.

Por el contrario, centrándonos en estudios con objetivos más próximos al de nuestra investigación, Ibarraran, Lasagabaster y Sierra (2008) muestran que los estudiantes de educación secundaria inmigrantes en el País Vasco tienen actitudes positivas hacia el español y hacia el inglés, pero mucho menos positivas hacia el vasco; situación que contrasta con los estudiantes autóctonos que mantienen actitudes más positivas y equilibradas hacia las tres lenguas. En el mismo contexto, Rojo, Madariaga y Huguet (2010) sitúan a los estudiantes de origen hispanoamericano como los menos receptivos hacia la lengua vasca.

Rojo, Huguet y Janés (2005) llevaron a cabo un estudio comparado de las actitudes lingüísticas del alumnado autóctono e inmigrante en Cataluña y Euskadi, en el que se constatan actitudes globalmente positivas hacia ambas lenguas, aunque es necesario precisar que son muy superiores hacia el catalán en comparación con el vasco. Por su parte, Madariaga, Huguet y Lapresta (2013) estudian las actitudes, la presión social y la educación inclusiva en aulas catalanas con diversidad lingüística y cultural y concluyen que las actitudes del alumnado autóctono son más favorables hacia el catalán, mientras que el alumnado inmigran- 
te- sobre todo los hispanohablantes- muestra actitudes más positivas hacia el español. La edad de llegada y el tiempo de estancia, no obstante, suavizan estas diferencias y correlacionan con los logros de competencia lingüística en ambas lenguas.

La comunidad autónoma del Principado de Asturias, centro de nuestro estudio, no es ajena al estudio de las actitudes lingüísticas hacia el asturiano, la lengua vernácula, y el español, lengua común del estado español. En 1994, Llera-Ramo realizó el primer gran estudio sociolingüístico en Asturias que se repitió en el 2002 (Llera-Ramo y San Martín 2003), y de nuevo en 2017 (Llera-Ramo). En este último, se evidencia como los asturianos se identifican claramente con la lengua asturiana, la cual está muy presente en las relaciones sociales más cercanas, cediendo espacio ante el castellano a medida que el vínculo personal es menor. Más reciénteme, Hevia (2018) presenta una investigación centrada en las opiniones de la juventud con la lengua asturiana, en donde se concluye que la juventud asturiana tiene actitudes lingüísticas especialmente positivas con respecto a las posibilidades de futuro de esta lengua. En el ámbito educativo, González (2001) estudia las actitudes lingüísticas de los estudiantes de secundaria autóctonos (14 años) con respecto a ambas lenguas y concluye que las actitudes son, globalmente, muy favorables a ambas lenguas, si bien con mayor puntuación cuando se trata del castellano. Louzao y González (2009), por su parte, plantean un estudio de caso para estudiar las actitudes interculturales del alumnado de educación primaria (12 años), del que se deriva que en determinados centros educativos los alumnos de origen inmigrante y sus familias manifiestan que el aprendizaje de la lengua propia de la comunidad constituye una oportunidad para mejorar su integración social y cultural en Asturias.

Finalmente, González, Hevia y Fernández (2013) realizan un estudio (solamente con alumnado autóctono) centrado en una pequeña parte del territorio asturiano y referido a la modalidad lingüística conocida como 'gallego-asturiano'2 y castellano. Las conclusiones establecen que las actitudes hacia ambas lenguas son muy positivas, si bien más en el caso del castellano. Del estudio se deriva, además, que los alumnos que tienen como lengua familiar al gallego-asturiano tienen mejores actitudes hacia ella, igual que los que reciben enseñanza de esta lengua.

Pues bien, a partir de las referencias previas, parece evidente la oportunidad de estudiar las actitudes lingüísticas hacia las lenguas en Asturias, pero considerando no solo al alumnado autóctono, sino haciendo la primera aproximación a las opiniones y actitudes del alumnado inmigrante del último curso de Educación Primaria. 


\section{Diseño metodológico}

\subsection{Participantes}

El asturiano como materia de estudio es elegido por más del 50\% de la población escolarizada en la Educación Primaria de los centros públicos. Por otro lado, nos encontramos con que la distribución del alumnado inmigrante en los centros educativos de la región es muy desigual. Como observamos en la Tabla 1, la asistencia a clases de lengua asturiana por parte del colectivo inmigrante se corresponde, grosso modo, con su presencia social a nivel general.

\begin{tabular}{|l|c|c|}
\hline \multicolumn{3}{|c|}{$\begin{array}{c}\text { Datos de escolarización en la Educación Primaria (6-12 años) de Asturias en Centros Públicos } \\
\text { Cur/2016 }\end{array}$} \\
\hline & Total & Alumnado extranjero \\
\hline Alumnado & 34.011 & $1.421(4,18 \%)$ \\
\hline $\begin{array}{l}\text { Alumnado que asiste a clases de asturiano en toda la etapa } \\
\text { educativa }\end{array}$ & 17.607 & $530(3,01 \%)$ \\
\hline Alumnado en 6 ${ }^{0}$ curso & 5.392 & $301(5,58 \%)$ \\
\hline Alumnado que cursa asturiano en 6 ${ }^{\circ}$ curso & 2.821 & $106(3,76 \%)$ \\
\hline
\end{tabular}

Tabla 1. Datos de escolarización en Educación Primaria. Fuente:

Consejería de Educación del Gobierno de Asturias (2015)

Dada la dispersión del alumnado inmigrante por los distintos centros de Educación Primaria de la región, se han seleccionado cuatro centros educativos con el mayor porcentaje de alumnado inmigrante asistente a clases de Lengua Asturiana durante el curso 2015/2016. ${ }^{3}$ Se trata de cuatro colegios ubicados en distintos ámbitos: dos de carácter urbano, uno de carácter semi-urbano y, finalmente, uno de carácter rural. Las proporciones de alumnado inmigrante en los centros oscilan entre el 13,79\%, en el caso mínimo, y el 46,67\%, en el caso máximo. Se constituye, de esta forma, una muestra intencional de 123 estudiantes de $6^{\circ}$ curso de Educación Primaria participantes en nuestro estudio. De ellos, 79 son alumnos autóctonos y 44 de origen inmigrante. El 66,7\% (82 estudiantes) asisten a clase de Lengua Asturiana, de los cuales un 26,8\% son de origen inmigrante (22 estudiantes). Respecto a la lengua familiar, algo más de la mitad (52\%) utilizan siempre el castellano, seguidos por los que se consideran bilingües (asturiano y castellano) (30,1\%). Después estarían los hogares en donde se habla una lengua extranjera (ruso, rumano, portugués, ucraniano, polaco, árabe, chino, lituano e inglés), que suponen un $17,9 \%$. Hay que destacar, por otro lado, que los estudiantes inmigrantes de habla hispana tienen tendencia a responder 
'castellano' cuando se les pregunta por la lengua familiar. Es preciso señalar, finalmente, que la situación socioprofesional de las familias de los alumnos se caracteriza por su adscripción ampliamente mayoritaria al nivel medio-bajo.

En la Tabla 2 se puede ver un resumen de la distribución de los escolares en cada uno de los centros en donde se realizó la recogida de información.

\begin{tabular}{|c|c|c|c|c|}
\hline \multicolumn{5}{|c|}{ Distribución de la muestra del estudio } \\
\hline Centro & Género & $\begin{array}{l}\text { Asistencia a } \\
\text { clase de } \\
\text { Lengua } \\
\text { Asturiana }\end{array}$ & Lengua familiar & Tipo de alumnado \\
\hline Centro 1 & $\begin{array}{l}\text { Niño: } 15(60 \%) \\
\text { Niña: } 10(40 \%)\end{array}$ & $\begin{array}{l}\text { Sí: } 25(100 \%) \\
\text { No: } 0(0 \%)\end{array}$ & $\begin{array}{l}\text { Castellano: } 5(20 \%) \\
\text { Bilingüe**: } 16(64 \%) \\
\text { Extranjera: } 4(16 \%)\end{array}$ & $\begin{array}{l}\text { Autóctono: } 19(76 \%) \\
\text { Inmigrante: } 6(24 \%)\end{array}$ \\
\hline Centro 2 & $\begin{array}{l}\text { Niño: } 11(57,9 \%) \\
\text { Niña: } 8(42,1 \%)\end{array}$ & $\begin{array}{l}\text { Sí: } 14(73,7 \%) \\
\text { No: } 5(26,3 \%)\end{array}$ & 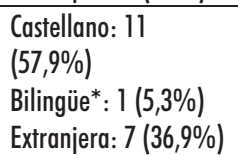 & $\begin{array}{l}\text { Autóctono: } 5(26,3 \%) \\
\text { Inmigrante: } 14 \\
(73,7 \%)\end{array}$ \\
\hline Centro 3 & $\begin{array}{l}\text { Niño: } 23(57,5 \%) \\
\text { Niña: } 17(42,5 \%)\end{array}$ & $\begin{array}{l}\text { Sí: } 16(40 \%) \\
\text { No: } 24(60 \%)\end{array}$ & 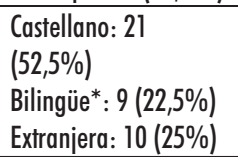 & $\begin{array}{l}\text { Autóctono: } 20(50 \%) \\
\text { Inmigrante: } 20(50 \%)\end{array}$ \\
\hline Centro 4 & $\begin{array}{l}\text { Niño: } 20(51,3 \%) \\
\text { Niña: } 19(48,7 \%)\end{array}$ & $\begin{array}{l}\text { Sí: } 27(69,2 \%) \\
\text { No: } 12(30,8 \%)\end{array}$ & 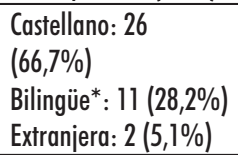 & $\begin{array}{l}\text { Autóctono: } 35 \\
(89,7 \%) \\
\text { Inmigrante: } 4 \\
(10,3 \%)\end{array}$ \\
\hline TOTAL & $\begin{array}{l}\text { Niño: } 69(56,1 \%) \\
\text { Niña: } 54(43,9 \%)\end{array}$ & $\begin{array}{l}\text { Sí: } 82(66,7 \%) \\
\text { №: } 41(33,3 \%)\end{array}$ & $\begin{array}{l}\text { Castellano: } 63 \\
(51,3 \%) \\
\text { Bilingïe*: } 37(30 \%) \\
\text { Extranjera: } 23 \\
(18,7 \%)\end{array}$ & $\begin{array}{l}\text { Autóctono: } 79 \\
(64,2 \%) \\
\text { Inmigrante: } 44 \\
(38,5 \%)\end{array}$ \\
\hline
\end{tabular}

*Bilingüe: hablan asturiano y castellano.

Tabla 2. Características del alumnado participante en el estudio por centro educativo

\subsection{Instrumento}

En la recogida de información se ha optado por utilizar el modelo de encuesta sociolingüística elaborado por el Servei d'Ensenyament del Catalá (Gabinet d'Estudis del SEDEC 1983) en base al trabajo de Sharp et al. (1973) y que ya ha demostrado su valía en contextos bilingües de España como Aragón (Huguet, Lapresta y Madariaga 2008), Cataluña (Huguet y Llurda 2001), País Vasco (Rojo, Mandariaga y Huget 2010) y el Principado de Asturias (González, Hevia y Fernández 2013). Además, este modelo ha sido probado en contextos de inmigración, 
obteniendo una tasa de fiabilidad satisfactoria a través de la técnica testretest, con índices de correlación $\mathrm{r}=0.703(\mathrm{p}<.0001)$ para el grupo inmigrante, $r=0.805(\mathrm{p}<.0001)$ para el grupo autóctono y $r=0.791(\mathrm{p}$ $<.0001$ ) para ambos grupos combinados (Huguet y Janés 2005). Se ha mantenido la misma estructura interna del instrumento a excepción de la inclusión del ítem 4 que permite controlar la asistencia o no a clase de Lengua Asturiana y la modalidad de respuesta 'Otra lengua' en aquellas preguntas referidas al uso lingüístico de los estudiantes, de sus familiares y de otras amistades.

De este modo, los criterios o parámetros de estudio se incluyen en el cuestionario que recoge aspectos relativos a datos personales (sexo, edad, lugar de nacimiento y de residencia), datos de escolarización (curso académico y tiempo en el colegio), profesión de los progenitores, lengua utilizada para comunicarse en el ámbito familiar y la asistencia o no a clases de lengua asturiana. Además de estos datos, se recogen las actitudes ante la coexistencia de lenguas a través de veinte ítems de respuesta dicotómica (sí/no) distribuidos al azar: 10 ítems se refieren a actitudes hacia el asturiano y 10 al castellano. ${ }^{4}$ Las preguntas se centran en evaluar las actitudes hacia estos dos idiomas indagando acerca de la opinión de los escolares sobre la pertinencia de que se estudie el asturiano, la utilidad del castellano y el asturiano o su relevancia y reconocimiento social, entre otras. Las puntuaciones totales para cada lengua nos permiten ubicar a cada estudiante en una de las tres categorías de actitud: favorable, neutra y desfavorable. Para la asignación de estas categorías se le da un valor de +1 por cada respuesta a favor de una lengua y -1 por las desfavorables. Posteriormente se realiza la suma algebraica de todos los valores, cuyo resultado puede variar entre +10 (actitud completamente favorable) y -10 (actitud completamente desfavorable). Con la puntuación total se encuadra a cada estudiante en una de las tres categorías descritas de acuerdo con el siguiente criterio: actitud favorable entre $+6 y+10$, neutra entre -5 y +5 y desfavorable entre -6 y -10 . Para determinar la fiabilidad de la prueba se empleó el Alfa de Cronbach, para la consistencia interna, la cual se sitúa en 0,87, mostrando un buen nivel de homogeneidad entre los ítems de la encuesta. Es preciso señalar, finalmente, que la justificación teórica de la anterior estructuración del cuestionario y su focalización temática tiene que ver con la posibilidad de contrastar sus resultados con los de estudios similares llevados a cabo tanto a nivel español como asturiano.

\subsection{Procedimiento}

El tratamiento estadístico de los datos se realizó a través del paquete estadístico IBM SPSS versión 22 para Windows. Además del análisis 
descriptivo de frecuencias, medidas de tendencia central y dispersión, para estudiar si las actitudes hacia la lengua castellana (ACT CAST) y hacia la lengua asturiana (ACT AST) difieren, se realizan las comparaciones de medias mediante pruebas paramétricas (Análisis de varianza univariante) y no paramétricas (prueba U de Man - Whitney y $\mathrm{H}$ de Kruskal-Walis), en función de la distribución en la variable criterio (asimetría y curtosis), si se cumple el principio de homocedasticidad (igualdad de varianzas en la variable criterio), teniendo en cuenta el tamaño y la diferencia en número de participantes en cada uno de los grupos estudiados (autóctonos e inmigrantes).

\section{Resultados y discusión}

Ofrecemos a continuación una síntesis de los principales resultados del estudio, tanto desde el punto de vista global como teniendo en cuenta las variables implicadas en la investigación. Las variables consideradas en este estudio, además de las de sexo, fueron las propias de los estudios sociolingüísticos que estudian las actitudes en contextos bilingües. Estas variables quedan descritas a continuación:

(1) Condición Lingüística Familiar (CLF): hace referencia a la lengua familiar del alumnado y se divide en tres categorías: castellano, bilingüe (asturiano y castellano) y lengua extranjera.

(2) Opcionalidad (OPC): se relaciona con la asistencia o no a las clases de lengua asturiana. Se contemplan, lógicamente, dos alternativas: Sí y No.

3) Procedencia del alumnado (PRO): este criterio permitirá analizar los resultados a partir del origen inmigrante o autóctono del alumnado.

\subsection{Resultados generales}

Los ítems implicados en la determinación de las actitudes hacia el asturiano y el castellano ofrecen un primer análisis de las opiniones de los estudiantes. Así, observamos que las actitudes son ampliamente favorables (65,9\% hacia el asturiano y $72,4 \%$ castellano), si bien es cierto que las actitudes hacia el castellano son 6 puntos más positivas. El porcentaje de actitudes neutras también es importante en ambos casos (30,9\% con respecto al asturiano y $27,6 \%$ en relación al castellano). Hay que decir que las actitudes desfavorables son mínimas y tan solo aparecen en el caso del asturiano (3,2\%). Véase Gráfico 1. 


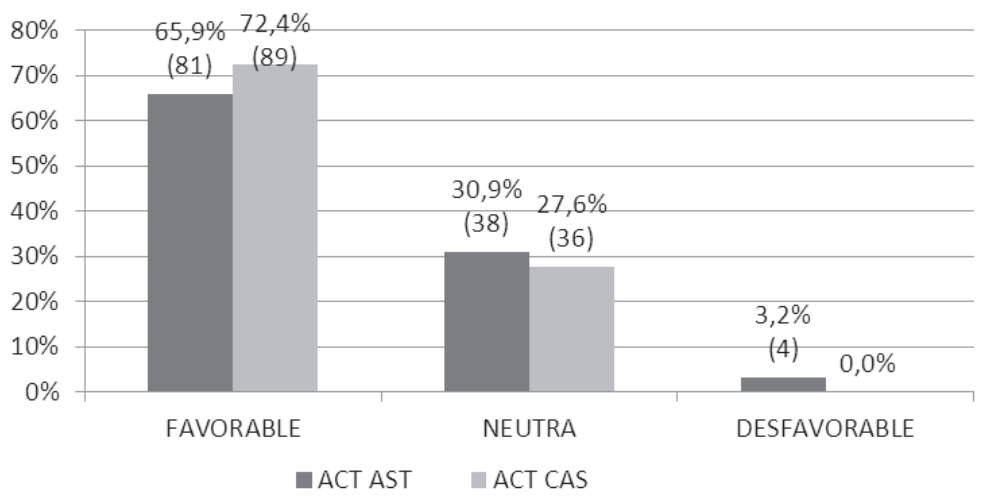

Gráfico 1: Actitudes hacia el Asturiano (ACT AST) y Actitudes hacia el Castellano (ACT CAS) en el total de la muestra (datos en \%)

Si se consideran los datos según la procedencia del alumnado, en la Tabla 3 comprobaremos que el porcentaje de actitudes favorables hacia la lengua asturiana es mayor entre el alumnado autóctono $(72,1 \%)$ que el inmigrante $(54,5 \%)$. Los porcentajes de actitudes hacia el castellano son similares, si bien es cierto que el del alumnado inmigrante $(72,7 \%)$ es unas décimas superiores al autóctono $(72,2 \%)$.

\begin{tabular}{|c|l|c|c|c|}
\hline Lengua & \multicolumn{1}{|c|}{ Actitud } & Autóctono & Extranjero & Total \\
\hline \multirow{3}{*}{ Castellano } & Favorable & $52(72,2 \%)$ & $32(72,7 \%)$ & $89(72,4 \%)$ \\
\cline { 2 - 5 } & Neutra & $22(27,8 \%)$ & $12(27,3 \%)$ & $34(27,6 \%)$ \\
\cline { 2 - 5 } & Desfavorable & $0 \%$ & $0 \%$ & $0 \%$ \\
\hline \multirow{3}{*}{ Asturiano } & Favorable & $57(72,1 \%)$ & $24(54,5 \%)$ & $81(65,9 \%)$ \\
\cline { 2 - 5 } & Neutra & $19(24,1 \%)$ & $19(43,2 \%)$ & $38(30,9 \%)$ \\
\cline { 2 - 5 } & Desfavorable & $3(3,8 \%)$ & $1(2,3 \%)$ & $4(3,2 \%)$ \\
\hline
\end{tabular}

Tabla 3. Actitudes hacia el castellano (ACT CAS) y al asturiano (ACT AST) del alumnado según procedencia

De la tabla anterior se deduce que las actitudes neutras hacia la lengua castellana son similares en ambos grupos y no llegan al 30\%, mientras que en el caso del asturiano el alumnado inmigrante presenta mayor porcentaje de posiciones neutras $(43,2 \%)$, bajando ligeramente el porcentaje en el caso del alumnado autóctono $(24,1 \%)$. En cuanto a las actitudes desfavorables, no identificamos opiniones negativas hacia la lengua castellana, pero sí mínimamente hacia la lengua asturiana, y es el alumnado autóctono quien presenta unas actitudes más desfavorables hacia la misma (3,8\% frente a $2,3 \%)$.

En síntesis, constatamos que las actitudes, tanto hacia el asturiano como hacia el castellano, son globalmente positivas. Es preciso aclarar 
que en el caso del castellano se registraron porcentajes más altos, aunque los porcentajes hacia el asturiano son también altos. Las actitudes neutras son también importantes (aunque se sitúan a una considerable distancia de las anteriores). En este sentido es el alumnado inmigrante el que en mayor medida manifiesta estas actitudes, sobre todo por lo que hace referencia al asturiano. Es preciso subrayar el hecho de que apenas existen actitudes negativas. Estos resultados van en la misma línea que los obtenidos por Rojo, Huguet y Janés (2005) al estudiar las actitudes lingüísticas del alumnado autóctono e inmigrante en Euskadi y Cataluña.

\subsection{Análisis de las variables explicativas de las actitudes lingüísticas}

En un primer momento se ha considerado necesario tener en cuenta la variable sexo, a fin de comprobar si se traslada o no a nuestro estudio el tradicional apego femenino por las formas y variedades lingüísticas de prestigio (López-Morales 1989). A tal efecto, hemos aplicado la prueba U de Mann-Whitney, tomando como variable de agrupación el sexo del alumnado que compone la muestra. Esta prueba pone de manifiesto que se establecen diferencias estadísticamente significativas entre los niños $(n=69)$ y las niñas $(n=54)$ en las actitudes hacia la lengua asturiana $(U=$ 1205,500; $\mathrm{p}=0,001$ ), y que son las niñas las que muestran unas actitudes más favorables hacia la lengua local. Se procedió a dar un paso más y se comprobó, comparando al alumnado por origen (inmigrante y autóctono), que mientras que en el alumnado inmigrante no hay diferencias por sexo en relación a las actitudes hacia ambas lenguas ( $p>0,05$ ), en el alumnado autóctono las niñas mantienen actitudes más favorables hacia la lengua asturiana $(U=440,000 ; p=0,001)$. Es decir, se reafirmarían aquí los resultados señalados por Serrano (2008) con respecto al menor conservadurismo de las mujeres en el contexto europeo.

Por otro lado, y con el propósito de seguir dando respuesta al segundo de los objetivos de la investigación, se ha realizado un análisis de las otras variables que pueden explicar las actitudes hacia la lengua castellana (ACT CAS) y la lengua asturiana (ACT AST) por parte de los escolares que participan en el estudio: asistencia a clase de Lengua Asturiana (OPC), condición lingüística familiar (CLF) y procedencia del alumnado (PRO).

En el caso de la primera de estas variables, el análisis de los datos muestra que la asistencia a clases de Lengua Asturiana (OPC) está profundamente relacionada con las actitudes lingüísticas de los escolares. Así, el alumnado que asiste a clases de Lengua Asturiana muestra acti- 
tudes más favorables al asturiano $(\mathrm{M}=6,82 ; \mathrm{D} . \mathrm{T}=3,120)$ que aquellos escolares que no asisten $(M=2,20 ; D . T=5,269)$, y estas diferencias son estadísticamente significativas $(U=736,500 ; p<0,001)$, tal como recogemos en la Tabla 4. En otras palabras, las actitudes hacia el asturiano de los escolares participantes en el estudio se relacionan inequívocamente con el hecho de cursar la asignatura de Lengua Asturiana.

\begin{tabular}{|l|c|c|c|c|c|}
\hline & $\begin{array}{c}\text { Asisten a clase de } \\
\text { Lengua Asturiana } \\
\mathbf{n = 8 2} \text { Media }\end{array}$ & D.T. & $\begin{array}{c}\text { No asisten a clase de } \\
\text { Lengua Asturiana } \\
\mathbf{n = 4 1 ~ M e d i a ~}\end{array}$ & D.T. & $\begin{array}{c}\text { U Mann- } \\
\text { Whitney }\end{array}$ \\
\hline ACT. AST & 6,82 & 3,120 & 2,20 & 5,269 & $736,500^{*}$ \\
\hline ACT.CAS & 6,18 & 3,374 & 6,66 & 3,111 & 1551,500 \\
\hline
\end{tabular}

* Las diferencias de rangos promedio son significativas al nivel $\mathrm{p}<0.001$ (bilateral).

Tabla 4. Comparación de actitudes favorables hacia el asturiano (ACT AST) y el castellano (ACT CAS) de todo el alumnado en función de asistencia a clase de Lengua Asturiana (OPC)

Además, tal como observamos en el Gráfico 2, los estudiantes que asisten a clase de Lengua Asturiana también manifiestan actitudes positivas hacia el castellano, aunque con una puntuación media ligeramente inferior $(\mathrm{M}=6,18 ; \mathrm{D} . \mathrm{T}=3,374)$. En este último caso (ACT CAST), se aprecia que las actitudes son similares independientemente de cursar Lengua Asturiana.

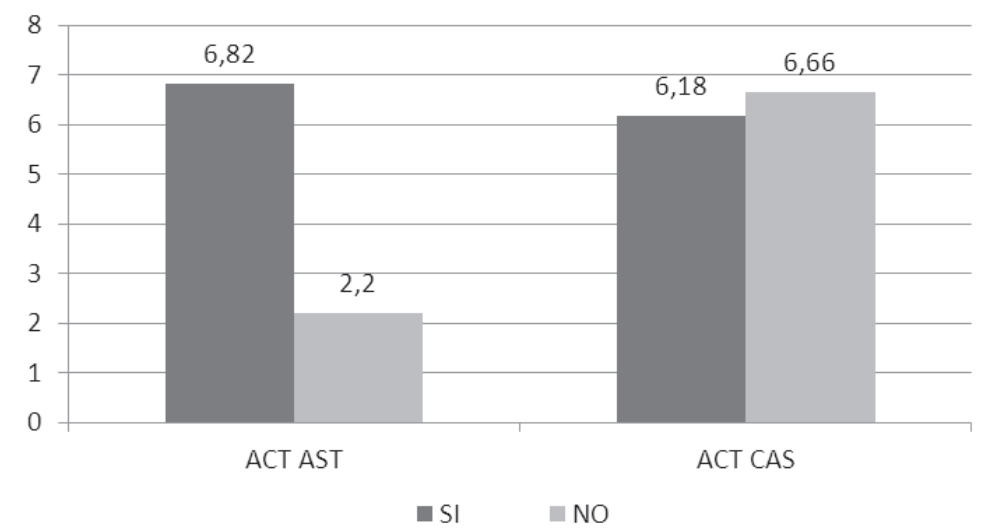

Gráfico 2. Medias de las actitudes favorables hacia el asturiano ( $A C T$ AST) y el castellano (ACT CAS) de todo el alumnado en función de la asistencia a clase de Lengua Asturiana (OPC) 
Respecto a la influencia de la condición lingüística familiar (CLF) en las actitudes lingüísticas, nos encontramos que la opinión más favorable hacia la Lengua Asturiana (ACT AST) la tienen quienes hablan en su entorno familiar las dos lenguas, es decir, los bilingües de asturiano y castellano (Media = 7,68; D.T = 2,801), siendo los estudiantes en cuyas familias se usa como medio de comunicación el castellano $(\mathrm{M}=4,25$; D. $T=4,772)$ o una lengua extranjera $(M=4,05 ; D . T=4,685)$ quienes muestran una actitud más desfavorable hacia la lengua asturiana. En definitiva, los escolares que usan la lengua asturiana en su vida familiar (aunque sea simultáneamente con el castellano) muestran actitudes más favorables hacia la misma.

En el caso de las actitudes hacia la lengua castellana (ACT CAS), nos encontramos que los estudiantes que en su hogar hablan una lengua extranjera son quienes presentan una puntuación media más elevada $(M=7,09 ; D . T=2,599)$, por encima de aquellos estudiantes que en su hogar hablan en castellano $(M=6,30 ; \mathrm{D} . \mathrm{T}=3,509)$ o son bilingües (asturiano y castellano) $(M=5,97 ; D . T=3,259)$. Estos datos se ilustran en el Gráfico 3.

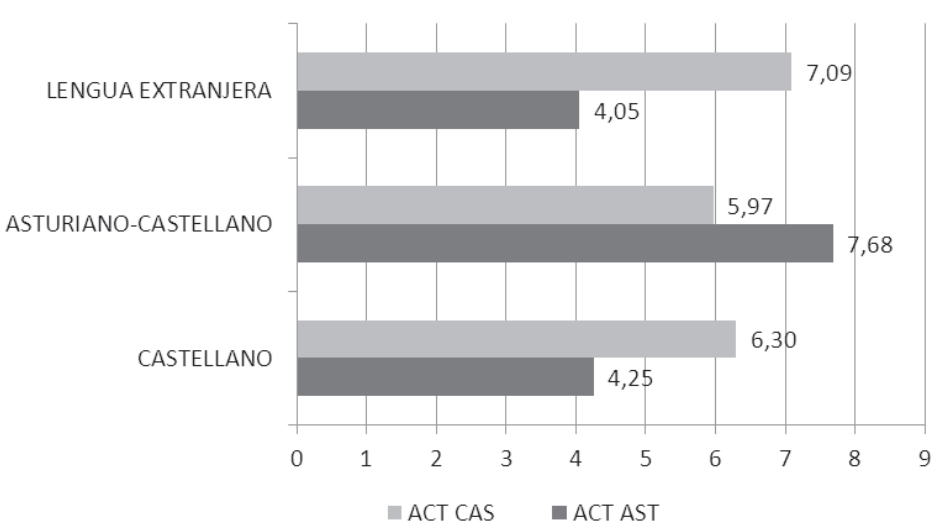

Gráfico 3. Medias de las actitudes favorables hacia el asturiano (ACT AST) y el castellano (ACT CAS) de todo el alumnado en función de la condición lingüistica familiar (CLF)

Apreciamos que el alumnado cuya condición lingüística familiar es una lengua extranjera, presenta puntuaciones medias superiores en ACT CAS que el alumnado que tiene como lengua de uso familiar el castellano o castellano y asturiano (bilingüe). El deseo de integración social y cultural a partir de la lengua mayoritaria y de prestigio podría explicar estos resultados.

Para la comparación entre los tres grupos aplicamos la prueba no paramétrica Kruskal-Wallis con el fin de determinar la existencia o no de dife- 
rencias significativas en cuanto a la actitud y la condición lingüística familiar. La prueba nos presenta diferencias significativas solamente en las actitudes hacia la lengua asturiana $\left(\chi^{2}=16,790 ; p=0,000\right)$ y, según los rangos promedio, los estudiantes que muestran unas actitudes más positivas son aquellos cuya condición lingüística familiar es bilingüe (asturiano y castellano), mientras que los estudiantes cuya lengua familiar es una lengua extranjera son quienes manifiestan opiniones menos favorables.

La tercera de las variables estudiadas tiene que ver con la procedencia del alumnado (PRO). En el caso del alumnado de origen inmigrante, este muestra una actitud más favorable hacia el castellano (Media= 6,39 y D.T.= 3,013) que hacia el asturiano (Media $=4,30$ y D.T.= 4,338). Por lo que respecta al alumnado autóctono, las actitudes están más equilibradas; así la opinión más favorable es hacia la lengua castellana (Media= 6,32 y D.T.= 3,444), opinión que sigue siendo favorable en el caso de las actitudes hacia el asturiano, si bien desciende ligeramente (Media = 5,82 y D.T.= 4,540). Hemos aplicado, al igual que en los casos anteriores, la prueba U de Mann-Whitney, tomando como variable de agrupación la procedencia del alumnado y se ha detectado que en el caso de la variable actitudes hacia el asturiano las diferentes opiniones sí alcanzan la significación estadística $(U=1307,000 ; p<0,05)$, siendo el alumnado autóctono el que presenta unas actitudes más favorables hacia la lengua asturiana, tal como se recoge en la Tabla 5.

\begin{tabular}{|l|c|c|c|c|c|}
\hline & $\begin{array}{c}\text { Alumnado de origen } \\
\text { extranjero } \mathbf{n = 4 4} \text { Media }\end{array}$ & D.T. & $\begin{array}{c}\text { Alumnado } \\
\text { autóctono } \\
\mathbf{n = 7 9} \text { Media }\end{array}$ & D.T. & $\begin{array}{c}\text { U Mann- } \\
\text { Whitney }\end{array}$ \\
\hline ACT. AST & 4,30 & 4,338 & 5,82 & 4,540 & $1307,000^{*}$ \\
\hline ACT. CAS & 6,39 & 3,013 & 6,32 & 3,444 & 1684,000 \\
\hline
\end{tabular}

* Las diferencias de rangos promedio son significativas al nivel $\mathrm{p}<0.05$ (bilateral).

Tabla 5. Comparación de actitudes favorables en función de la procedencia del alumnado (extranjero/autóctono).

A partir de estos resultados -los primeros obtenidos en Asturias en relación al alumnado de origen inmigrante escolarizado en esta comunidad autónoma- podemos plantear unas primeras conclusiones que posteriores estudios (y con muestras más amplias) habrán de confirmar o desmentir.

\section{Conclusiones}

Hemos estudiado las actitudes de una muestra de estudiantes autóctonos e inmigrantes hacia las lenguas habladas en la comunidad asturiana 
(castellano y asturiano) constatando, en primer lugar, que los participantes en el estudio manifiestan actitudes lingüísticas globalmente positivas hacia ambas lenguas, si bien con porcentajes más altos en el caso del castellano. Aunque nos encontramos en un contexto en donde existe una lengua minoritaria sin reconocimiento oficial y ante la que existen ciertos prejuicios lingüísticos en algunos sectores de población, el alumnado muestra un interés por la lengua local, un interés que podría entenderse en función de los mecanismos que toda lengua minoritaria pone en juego para generar opiniones favorables a su supervivencia (Ehala y Niglas 2006; Wright y Bougie 2007).

Al estudiar los resultados obtenidos en función del origen del alumnado, comprobamos que estas valoraciones adquieren matices cuando entran en comparación, y que están condicionadas por las identidades sociales de cada grupo. Así, en el caso del alumnado autóctono las actitudes son positivas y equilibradas hacia las dos lenguas, mientras que en el caso del alumnado inmigrante las actitudes también son positivas, pero menos equilibradas (en el caso del asturiano, en mucha menor medida). Estos resultados serían similares a los obtenidos en otras comunidades bilingües de España, por ejemplo, el País Vasco (Ibarraran, Lasagabaster y Sierra 2008) o Cataluña (Madariaga, Huguet y Lapresta 2013). No obstante, a diferencia de esos otros contextos de estudio, estamos hablando de una lengua especialmente minoritaria por lo que las actitudes positivas del alumnado inmigrante pueden jugar un papel importante para establecer el componente simbólico que, al margen del castellano, desempeña el asturiano en las relaciones que se establecen entre la comunidad inmigrante y la receptora.

Por otro lado, destacan las diferencias actitudinales en relación a la variable sexo en el caso de la lengua asturiana. Este resultado, en concordancia con la literatura científica (Trudgill 1992; Serrano 2008; Romaine 1994), evidencia el importante papel desempeñado por el sexo en los estudios sociolingüísticos. En este caso, como hemos señalado, se verifica el planteamiento de Serrano (2008) según el cual las mujeres de diferentes comunidades dan soluciones distintas a la participación en el cambio lingüístico, y se refuta la teoría de Trudgill (1992) según la cual las mujeres tienen una tendencia más positiva hacia la variedad de más prestigio. No tenemos constancia de otras investigaciones en nuestro entorno en las que, como en la presente, se ponga de manifiesto que las niñas mantienen actitudes más favorables hacia la lengua regional minoritaria y, menos aún, que sean las niñas autóctonas y no las extranjeras las responsables de tales diferencias; aunque sí se puede comprobar que en el contexto internacional la variable 'sexo' del alumnado sí puede tener relevancia ocasional en este tipo de estudios (Heining-Boynton y Haitema 2007). Es por ello que sería interesante, en este sentido, un 
estudio más amplio que ahondase en esta cuestión puesto que, como hemos visto, el argumento del prestigio, por sí solo, es incapaz de explicar un fenómeno tan complejo como la variación lingüística ligada al sexo en este contexto de estudio.

También observamos que la asistencia a clases de lengua asturiana, así como el hecho de que el asturiano sea usado en el medio familiar, son factores relacionados con actitudes favorables hacia el asturiano y también hacia el castellano. Por el contrario, como era de esperar, el hecho de no recibir clases de asturiano y de tener como lengua familiar el castellano o una lengua extranjera son factores que se relacionan con actitudes menos favorables hacia el asturiano. Los resultados parecen confirmar, en este sentido, los datos de otros estudios previos realizados en Asturias (González 2001; González, Hevia y Fernández 2013).

Es preciso señalar, finalmente, que el presente estudio (de carácter descriptivo e introductorio) debería complementarse en el futuro con otros que tuvieran una base empírica o muestra más amplia y que contase, además, con técnicas complementarias de carácter cualitativo (entrevistas en profundidad y grupos de discusión, entre otras). A tal efecto, el equipo de investigación trabaja en un diseño de un proyecto específico que espera llevar a la práctica próximamente.

Isabel Hevia Artime

Universidad de Oviedo

heviaisabel@uniovi.es

ORCID: 0000-0001-7446-2601

Patricia Albá García

Universidad de Oviedo

patriciagarcia@uniovi.es

Recepción: 26/07/2018; Aceptación: 05/10/2019

\author{
Xosé Antón González Riaño \\ Universidad de Oviedo \\ xanton@uniovi.es
}

ORCID: 0000-0001-6802-3389

\section{Notas}

1 El trabajo forma parte de una investigación más amplia financiada por el Ministerio de Economía y Competitividad de España (referencia nº EDU2014-54093-R) y la Agència de Gestió d'Ajuts Universitaris i de Recerca de la Generalitat de Catalunya (referencia $\mathrm{n}^{\circ}$ : 2014 SGR 208).

2 El gallego-asturiano es la variedad lingüística específica que se extiende por el espacio geográfico asturiano comprendido entre los ríos Navia y Eo. A menudo se la caracteriza como una lengua de transición en la que se mezclan características propias del gallego (comunidad lingüística limítrofe con Asturias) y del asturiano, junto con otros rasgos lingüísticos específicos de esta zona.

3 Nuestro más sincero agradecimiento a esas escuelas por su buena disposición y por su colaboración.

4 Ejemplo de ítems: Favorable asturiano: "Me parece bien que todos los niños y niñas de Asturias estudien asturiano"; Desfavorable hacia el asturiano: "Es desagradable apren- 
der asturiano"; Favorable castellano: "El castellano debería enseñarse en todos los países"; Desfavorable castellano "El castellano sólo deberían aprenderlo y estudiarlo los que lo hablan".

\section{Referencias bibliográficas}

Allport, Gordon. 1935. “Attitudes”. En A Handbook of Social Psychology, ed. por Carl Murchison, 98-844. Worcester: Clark University Press.

Almeida, Manuel y Carmelo Vidal. 1995. "Variación socioestilística del léxico: un estudio contrastive". Boletín de Filología, 35. 49-65.

Appel, René y Pieter Muysken. 1996. Bilingüismo y contacto de lenguas. Barcelona: Ariel. Baker, Colin. 1992. Attitudes and Language. Clevedon: Multilingual Matters.

Breckler, Steven. 1984. "Empirical validation of affect, behavior and cognition as distinct components of attitudes". Journal of Personality and Social Psychology, 47:6. 1191- 1205.

Consejería de Educación y Ciencias del Principado de Asturias (2015). Alumnáu en Llingua Asturiana. (Informe inédito). Oviedo: Consejería de Educación y Ciencias del Principado de Asturias.

Ehala, Martin y Katrin Niglas. 2006. "Language Attitudes of Estonian Secondary School Students". Journal of Language, Identity, and Education, 5:3. 209-227.

Esteban, Maria del Carmen y Farah Mohamed Chaib. 2014. "El valor predictivo de las actitudes lingüísticas en la Educación Primaria en una comunidad de habla: El caso de los hablantes de dariya en Ceuta". Tonos digital: Revista electrónica de estudios filológicos, 26. 1-15.

García-Marcos, Francisco. 2015. Sociolingüística. Madrid: Síntesis.

Gardner, Robert y Peter MacIntyre. 1993. “A student’s contribution to Second Language Learning: Part II, Affective Factors”. Language Teaching, 26. 1-11.

González, Xosé Antón. 2001. "Actitúes sociollingüístiques del alumnáu de Secundaria n'Asturies”. Lletres Asturianes, 78. 7-27.

González, Xosé Antón. 2002. Manual de Sociollingüística. Uviéu: Academia de la Llingua Asturiana.

González, Xosé Antón, Isabel Hevia y Alberto Fernández. 2013. "Language Attitudes of Asturian Students in the Area of Navia-Eo". Language and Intercultural Communication, 13:4. 450-469.

Hamers, Josiane y Michel Blanc. 1983. Bilingualité et Bilinguisme. Bruxelles: Mardaga.

Heining-Boynton, Audrey y Thomas Haitema. 2007. "A Ten-year Chronicle of Student Attitudes towards Foreign Language in the Elementary School”. The Modern Language Journal, 91:2. 149-168.

Hevia, Isabel. 2018. Los jóvenes y la lengua asturiana. Avance de resultados. Oviedo: Academia de la Llingua Asturiana.

Huguet, Ángel. 2001. "Lenguas en contacto y educación: influencia del prestigio de las lenguas en las actitudes lingüísticas de los escolares". Revista de Educación, 326. 355-371.

Huguet, Ángel y Xosé Antón González. 2004. Actitudes lingüísticas, lengua familiar y enseñanza de la lengua minoritaria. Barcelona: Horsori.

Huguet, Ángel, Cecilio Lapresta y José María Madariaga. 2008. “A study on language attitudes towards regional and foreign languages by schoolchildren in Aragon, Spain”. International Journal of Multilingualism, 5:4. 275-293.

Huguet, Ángel y Enric Llurda. 2001. "Language Attitudes of School Children in Two Catalan/Spanish Bilingual Communities". International Journal of Bilingual Education and Bilingualism, 4:4. 267-282.

Huguet, Ángel y Judit Janés. 2005. "Niños inmigrantes en sociedades bilingües. Las actitudes ante las lenguas por parte de los escolares recién llegados a Cataluña". Cultura EEducación, 17:4. 309-31

Lengua y migración / Language and Migration 12:2 (2020), 87-108

Edición impresa: ISSN 1889-5425. Edición en línea: ISSN 2660-7166. @ Universidad de Alcalá 
Huguet, Ángel y Jordi Suils. 1998. Contacte entre llengües $i$ actituds lingüístiques. Barcelona: Horsori.

Ibáñez, Tomas (coord.). 2004. Introducción a la psicología social. Barcelona: Editorial UOC. Ibarraran, Amaia, David Lasagabaster y Juan Manuel Sierra. 2008. "Multilingualism and Language Attitudes: Local Versus Immigrant Students'Perceptions”. Language Awareness, 17:4. 326-341.

ICSLRA 2004. Informe y recomendaciones sobre la lengua asturiana. Lieja: International Committee for the Safeguarding of Linguistic Rights in Asturies/Association Internationale pour la Défense des Langues et Cultures Menacées.

INE (Instituto Nacional de Estadística). 2016. Continuos Register Statistics. Recuperado el 15 de abril de 2016 from http://www.ine.es

Janés, Judit. 2006. "Las actitudes hacia las lenguas y el aprendizaje linguístico". Revista Interuniversitaria de Formación del Profesorado, 20:2. 117-132.

Lasagabaster, David. 2003. Trilingüismo en la enseñanza. Actitudes hacia la lengua minoritaria, la mayoritaria y la extranjera. Lleida: Milenio.

Lewandowski, Theodor. 1982. Diccionario de lingüística. Madrid: Cátedra.

Labov, William. 1966. The Social Stratification of English in New York City. Cambridge: Cambridge University Press.

Ley 1/1998, de 23 de marzo, de uso y promoción del bable/asturiano. Boletín Oficial del Estado, 103, de 30 de abril de 1998, 14573 a 14576.

Llera-Ramos, Francisco. 2017. Los asturianos y la lengua asturiana. Estudio sociolingüístico para Asturias, 1991. Oviedo: Servicio de Publicaciones del Prinicpado de Asturias.

Llera-Ramos, Francisco y Pablo San Martín. 2003. II Encuesta Sociolingüística de Asturias. Oviedo: Academia de la Llingua Asturiana.

Llera-Ramos, Francisco. 2017. III Encuesta Sociolingüistica de Asturias (Avance de Resultados). Oviedo: Academia de la Llingua Asturiana.

López-Morales, Humberto. 1989. Sociolingüística. Madrid: Gredos.

Louzao, Maria y Xosé Antón González. 2009. “Investigación sobre les actitúes interculturales del alumnáu asturianu d'Educación Primaria. Un estudiu de casu”. Lletres Asturianes, 100. 107-126.

Madariaga, José María, Ángel Huguet y Cecilio Lapresta, C. 2013. “Actitud, presión social y educación inclusiva en aulas con diversidad lingüística y cultural”. Educación $X X 1,16: 1.305-328$.

Masgoret, Anne-Marie y Robert Gardner. 2003. “Attitudes, Motivation, and Second Language Learning: A Meta-analysis of Studies Conducted by Gardner and Associates". Language Learning, 53:1. 123-163.

Milroy, James. 2007. "The ideology of the standard language". En The Routledge Companion to Sociolinguistics, ed. por Carmen Llamas, Louise Mullany y Peter Stockwell, 133-139. Londres, Nueva York: Routledge.

Moreno, Francisco. 1998. Principios de sociolinguística y sociología del lenguaje. Barcelona: Ariel Lingüística.

Moriarty, Máiréad. 2010. The Effects of Language Planning Initiatives on the Language Attitudes and Language Practices of University Students. A Comparative Study of Irish and Basque". Language Problems and Language Planning, 34:2. 141-157.

Oskamp, Stuart. 1991. Attitudes and Opinion. Englewood Cliffs (NJ): Prentice Hall.

Paris, Django. 2011. Language across difference. Ethnicity, Communication, and Youth Identities in Changing Urban Schools. Cambridge: Cambridge University Press.

Pérez, Laura. 2016. "Las actitudes lingüísticas de los jóvenes hispanos de Montreal”. Lengua y Migración, 8:2. 105-132.

Perlman, Daniel y Christopher Cozby. 1985. Psicología Social. México: Interamericana.

Romaine, S. 1994. Language in Society. An Introduction to Sociolinguistics. Oxford: OUP.

Rojo, Vanesa, Ángel Huguet y Judit Janés. 2005. “Una aproximación a las actitudes lingüísticas del alumnado autóctono e inmigrante en Euskadi y Cataluña”. Revista de Psicodidáctica, 10:1. 75-83. 
Rojo, Vanesa, José María Madariaga y Ángel Huguet. 2010. "Las actitudes lingüísticas del alumnado autóctono e inmigrante de la educación secundaria en la comunidad autónoma vasca". Segundas Lenguas e Inmigración, 3. 43-60.

Sancho Pascual, María. 2013. "Identidad y prestigio en las actitudes lingüísticas de la población ecuatoriana en Madrid”. Lengua y Migración, 5:1. 33-56.

SEDEC (Servei d'Ensenyament del Català). 1983. Quatre anys de catalá a l'escola. Barcelona: Departament d'Ensenyament de la Generalitat de Catalunya.

Serrano, María José. 2008. "El rol de la variable sexo o género en sociolingüística: ¿diferencia, dominio o interacción?”. Boletín de Filología, 43:1. 175-192.

Schneiderman, Eta. 1976. "An Examination of the Ethnic and Linguistic Attitudes of Bilingual Children”. Review of Applied Linguistic, 33. 89-113.

Sharp, Derrick, Thomas Beryl, Price Eurwen, Francia Gareth y Davies Iwan. 1973. Attitudes to Welsh and English in the Schools of Wales. London: McMillan, University of Wales Press.

Sorolla, Ileana. 2011. La nueva Babel: la dimensión lingüística en la migración internacional. Centro de Estudios de Migraciones Internacionales, Universidad de La Habana.

Spencer, Herbert. 1862. First Principles. London: Williams and Norgate.

Tremblay, Paul y Robert Gardner. 1995. "Expanding the Motivation Construct in Language Learning”. Modern Language Journal, 79. 505-518.

Trudgill, Peter. 1985. Sociolinguistics. An Introduction to Language and Society. Britain: Penguin.

Trudgill, Peter. 1992. Introducing Language and Society. London: Penguin.

Trudgill, Peter y Jose Manuel Hernández. 2007. Diccionario de Sociolingüística. Madrid: Gredos.

Tusón, Jesús. 1995. Los prejuicios lingüísticos. Barcelona: Octaedro.

Verkuyten, Maykel y Jochem Thijs. 2010. "Ethnic Minority Labeling, Multiculturalism, and the Attitude of Majority Group Members". Journal of Language and Social Psychology, 29:4. 417-477.

Wallwork, Jean. 1985. Language and Linguistics. London: Heineman.

Wright, Stephen y Évelyne Bougie. 2007. "Intergroup Contact and Minority-language Education: Reducing Language-based Discrimination and its Negative Impact". Journal of Language and Social Psychology, 26:2. 157-181.

Zanna, Mark y John, Rempel. 1988. "Attitudes: a new look at an old concept". En The social psychology of knowledge, ed. por Daniel Bar-Tal y Arie Kruglanski, 315-334. Cambridge: Cambridge University Press.

Lengua y migración / Language and Migration 12:2 (2020), 87-108

Edición impresa: ISSN 1889-5425. Edición en línea: ISSN 2660-7166. () Universidad de Alcalá 